\title{
TAXONOMIA DE LOS HELMINTOS DE ALGUNOS ANFIBIOS DE LA CALERA [CUNDINAMARCA] *
}

\section{Planteamiento del problema}

El Departamento de Biología en su propósito de cumplir con una de los objetivos de UPN., está interesado en fomentar la investigación a nivel de estudiantes y profesores con el fin de integrar la labor docente e investigativa en la formación de los futuros profesionales de la educación. En respuesta a este estímulo del Departamento, y teniendo en cuenta que nuestro medio la investigación en este campo ha sido mínima, por no decir nula, hemos iniciado el presente trabajo. Al mismo tiempo nos proponemos aportar nuevos conceptos a los lectores de esta revista, en el campo de la parasitología y sus implicaciones e incidencia en la vida de los animales y el hombre.

Este trabajo puede ser la iniciación de posteriores investigaciones de mayor cobertura y será una contribución a otras instituciones interesadas en el estudio del balance ecológico para la conservación de los recursos naturales.

Trabajos similares han sido desarrollados en otros países, incluyendo algunos latinoamericanos tales como Argentina, Brasil, México, Ecuador, Perú, etc., destacándose el grupo de los anuros.

La mayoría de las investigaciones sobre helmintos se han realizado en vertebrados en general y especialmente en vertebrados domésticos.

La investigación está basada en los siguientes interrogantes:

1. ¿Hospedan los anfibios de Colombia las especies de Helmintos ya descritos por otros investigadores en este campo?

2. ¿Son éstas especies nuevas?

Como respuesta a los interrogantes formulados postulamos: si las especies que se encuentren son las mismas o semejantes a las ya descritas, entonces los helmintos pueden contribuir al esclarecimiento de la filogenesis de los anfibios en América.

\section{Objetivos}

1. Despertar el interés científico del estudiante para que participe en este tipo de investigaciones.

2. Identificar las especies de Helmintos que parasitan los anfibios de La Calera.

3. Coleccionar material didáctico para uso del departamento de Biología de la Universidad Pedagógica Nacional.

\footnotetext{
* Esta investigación esta a cargo de Profesores del Departamento de Biología.
} 
4. Establecer un intercambio permanente de materiales e información pertinente a esta área, con instituciones afines.

\section{Metodología}

Los anfibios se capturan a mano o mediante el uso de redes, en charcas y pozos de áreas rurales de La Calera. Los animales son traídos luego al laboratorio y disectados al día siguiente de su captura. El reconocimiento de las especies se hace en la Universidad Nacional —Instituto de Ciencias Naturales-. Los órganos internos (vísceras) son extraídos y colocados en una caja de petri pequeña que contiene una solución salina al $0.7 \%$.

El intestino y demás órganos son abiertas con ayuda de las agujas de disección. Los parásitos hallados en cada órgano son colocados en una cajita de petri diferente.

Los nemátodos se procesan. Se montan en gelatina y resma por el método de AlcoholGlicerina hirviendo; los tremátodos, céstodos y Acantocéfalos se fijan en A.F.A. (AlcoholFormol, Ácido Acético), se tiñen con acetocarmin, se deshidratan con etanol y se montan con una buena resina.

\section{Estado actual del proyecto}

Hasta el momento se han estudiado 101 especimenes de Hyla labialis labialis y 18 especimenes de Colestetus subpunctatus subpunctatus: Los Nemátodos hallados en Hyla labialis labialis pertenecen a los géneros Thelandros Wedl, 1861 y Dibulbiyer Caballero, 1935.

Los Tremátodos y Acantocéfalos hallados están aún sin clasificar.

Es de anotar que no se han encontrado Céstodos en estos anfibios estudiados.

\section{LA ENSEÑANZA DE LAS MATEMATICAS*}

\section{Planteamiento del Problema}

En el Departamento de Matemáticas siempre ha existido la preocupación por mejorar la calidad de la enseñanza en todos los niveles, en particular en el primario. Sin embargo, los esfuerzos que se han hecho en este sentido no han podido ser lo suficientemente sistemáticos y los resultados que han producido pueden apreciarse cualitativamente, pero no se han demostrado estadísticamente.

Se detecta que a pesar de las condiciones especiales del Instituto Pedagógico Nacional en muchos aspectos, siguen dándose los fenómenos habituales de rechazo y/o bajo rendimiento académico con respecto a las matemáticas.

\footnotetext{
* Investigación realizada por un grupo de profesores del Departamento de Matemáticas.
} 